\title{
Wormhole and its Analogue in Brane World
}

\author{
Subenoy Chakraborty* and Tanwi Bandyopadhyay \\ Department of Mathematics, Jadavpur University, Kolkata-32, India.
}

(Dated: November 21, 2018)

\begin{abstract}
In Einstein gravity, for an inhomogeneous phantom energy distribution having linear equation of state (but anisotropic), there exists simple exact solution for spherically symmetric space time describing a wormhole. At infinity, the space time is not asymptotically flat and possesses a regular cosmological Killing horizon with an infinite area. In this work, we have shown that, this wormhole solution is also possible in brane world for various matter distribution, which are not necessarily phantom in nature.

PACS numbers: $98.80 . C q, \quad 04.20 . G z, 04.50 .+h$
\end{abstract}

Wormholes are usually defined as smooth bridges between different universes or topological handles (i.e, throats, having no horizons) between remote parts of a single universe. Earlier, wormholes are purely of theoretical curiosity $[1,2]$, but recently this theoretical aspect has gained significant importance due to the present accelerating phase of our universe $[3,4]$. There is a nice similarity between this theoretical phenomenon and the recent observational aspects. A traversable wormhole is supported by so called exotic matter with a negative pressure $(p<0)$ and violation of the null energy condition (i.e, $\rho+p<0$ ) at least in a neighbourhood of the wormhole throat [5]. On the other hand, the observed acceleration of the universe is due to a hypothetical dark energy, violating the strong energy condition (i.e, $\rho+3 p<0$ ). A useful choice of dark energy is the phantom energy [6] having equation of state, $p=-\omega \rho, \omega>1$ (thereby violates null energy condition). However, there is one basic difference between the above two issuesin static wormhole, the matter distribution depends on the spatial coordinates while in cosmology, the matter density, pressure, are usually functions of temporal coordinates.

In recent years wormholes have been discussed in brane world scenario, an interesting concept in which, our universe is a 3-brane embedded in a five dimensional bulk $[7,8,9]$. Bronnikov and Kim [10] have obtained a class of static and spherically symmetric solutions in vacuum brane and bulk Weyl effects support the wormhole. Very recently, Lobo [11] has given a general formulation for brane world wormholes and has presented two possible wormhole configurations with dust and perfect fluid having linear equation of state, as the brane matter.

Here we shall show that, wormhole supported by phantom energy, as described by Zaslavskii [12], can be considered as a possible wormhole solution in brane scenario for various matter distribution in the brane and they are not necessarily violating the null energy conditions.

In Schwarzschild coordinates, the spherically symmetric metric describes that, traversable wormhole can be cast in the form $[13,14]$

$$
d s^{2}=-e^{2 \Phi} d t^{2}+\frac{d r^{2}}{1-\frac{b(r)}{r}}+r^{2} d \Omega_{2}^{2}
$$

where $\Phi(r)$ is called the redshift function and $b(r)$ stands for the shape function. In an orthonormal reference frame, the non vanishing components of the Einstein field equations are

$$
\begin{gathered}
\rho(r)=\frac{1}{8 \pi G} \frac{b^{\prime}}{r^{2}} \\
p_{r}(r)=\frac{1}{8 \pi G}\left[\frac{2}{r}\left(1-\frac{b(r)}{r}\right) \Phi^{\prime}-\frac{b}{r^{3}}\right] \\
p_{t}(r)=\frac{1}{8 \pi G}\left(1-\frac{b(r)}{r}\right)\left[\Phi^{\prime \prime}+\Phi^{\prime}\left(\Phi^{\prime}+\frac{1}{r}\right)\right] \\
-\frac{\left(b^{\prime} r-b\right)}{2 r^{2}}\left(\Phi^{\prime}+\frac{1}{r}\right)
\end{gathered}
$$

in which $\rho(r)$ is the energy density, $p_{r}(r)$ is the radial pressure and $p_{t}(r)$ is the lateral pressure. The conservation of the stress-energy tensor gives

$$
p_{r}^{\prime}=\frac{2}{r}\left(p_{t}-p_{r}\right)-\left(\rho+p_{r}\right) \Phi^{\prime}
$$

Let us suppose that our matter source is described by the phantom energy with equation of state [14]

$$
p_{r}=-\omega_{1} \rho \quad \text { and } \quad p_{t}=\omega_{2} \rho, \quad \omega_{1}>1
$$


Then Zaslavskii [12] has obtained a simple solution with the choice $\omega_{2}=\frac{\omega_{1}-1}{4}>0$ as

$$
\begin{gathered}
b=r_{0}+d\left(r-r_{0}\right), \quad e^{2 \Phi}=\frac{r_{1}}{r}, \quad \rho=\frac{d}{8 \pi r^{2}}, \\
p_{r}=-\frac{1}{8 \pi r^{2}}, \quad p_{t}=\frac{1-d}{32 \pi r^{2}}
\end{gathered}
$$

where $r_{0}$ (throat radius) and $r_{1}$ are arbitrary constants and $d=\frac{1}{\omega_{1}}<1$. Note that as $b^{\prime}\left(r_{0}\right)=d<1$, so flaring of the throat is satisfied. Further, if we demand as usual $\omega_{2} \leq 1$, then $\omega_{1}$ is restricted by the inequality

$$
1<\omega_{1} \leq 5
$$

The proper radial distance is related to the shape function by

$$
\begin{gathered}
l(r)= \pm \int_{r_{0}}^{r} \frac{d r^{\prime}}{\sqrt{1-\frac{b\left(r^{\prime}\right)}{r^{\prime}}}}= \pm \frac{1}{\sqrt{1-d}}\left\{\sqrt{r\left(r-r_{0}\right)}\right. \\
\left.+\ln \left[\sqrt{\frac{r}{r_{0}}}+\sqrt{\frac{r}{r_{0}}-1}\right]\right\}
\end{gathered}
$$

where ' \pm ' signs stand for upper and lower part of the wormhole or universe. The metric (1) is not asymptotically flat for the solution (7), rather it can be glued to the external Schwarzschild solution at some finite radial distance.

In Randall-Sundrum type-II brane model [8], the generalized Einstein equation on the brane has the form $[9,15$ and for review see 16]

$$
G_{\mu \nu}=\kappa^{2} T_{\mu \nu}+\frac{6 \kappa^{2}}{\lambda} \Pi_{\mu \nu}-\Lambda g_{\mu \nu}-\varepsilon_{\mu \nu}
$$

with $\Lambda=\frac{1}{2}\left(\Lambda_{5}+\kappa^{2} \lambda\right)$.

Here $\Lambda$ and $\Lambda_{5}$ are respectively the cosmological constants on the brane and bulk and $\lambda$ is the brane tension (vacuum energy). The matter confined to the brane is described by the energy-momentum tensor $T_{\mu \nu}$ (such that $T_{A B} n^{B}=0$ ) and the correction term $\Pi_{\mu \nu}$ (the local effects of the bulk arising from the brane extrinsic curvature) which is quadratic in $T_{\mu \nu}$, has the expression

$\Pi_{\mu \nu}=\frac{1}{12} T T_{\mu \nu}-\frac{1}{4} T_{\mu \alpha} T_{\nu}^{\alpha}+\frac{1}{8} g_{\mu \nu}\left(T_{\alpha \beta} T^{\alpha \beta}-\frac{1}{3} T^{2}\right)$
The other correction term $\varepsilon_{\mu \nu}$ denotes the nonlocal bulk effects and is the projection of the five dimensional Weyl tensor on the brane, i.e,

$$
\varepsilon_{\mu \nu}={ }^{(5)} C_{A B C D} n^{B} n^{D} \delta_{\mu}^{A} \delta_{\nu}^{C}
$$

with $\varepsilon_{\mu}^{\mu}=0$ (traceless).

Due to static and spherically symmetric nature of the wormhole metric (1), we consider an isotropic fluid on the brane, i.e,

$$
T_{\mu \nu}=\operatorname{diag}(\rho, p, p, p)
$$

where $\rho(r)$ is the energy density and $p(r)$ is the isotropic pressure. As a result, the nonlocal bulk effects contribute in the form of an effective anisotropic fluid as

$$
\varepsilon_{\mu \nu}=\operatorname{diag}\left[\epsilon(r), \sigma_{r}(r), \sigma_{t}(r), \sigma_{t}(r)\right]
$$

Thus the generalized Einstein equation (9) on the brane for the metric (1) with matter distribution given by (12) and (13), is the modified version of the equations (2)-(4), where the left hand sides viz. $\rho, p_{r}$ and $p_{t}$ are replaced by $\rho^{e f f}(r), p_{r}^{e f f}(r)$ and $p_{t}^{e f f}(r)$ respectively i.e, (choosing $8 \pi G=1$ )

$$
\begin{gathered}
\frac{b^{\prime}}{r^{2}}=\rho^{e f f}(r)=\rho\left(1+\frac{\rho}{2 \lambda}\right)-\frac{\epsilon}{8 \pi} \\
\frac{2}{r}\left(1-\frac{b}{r}\right) \Phi^{\prime}-\frac{b}{r^{3}}=p_{r}^{e f f}(r) \\
=p\left(1+\frac{\rho}{\lambda}\right)+\frac{\rho^{2}}{2 \lambda}-\frac{\sigma_{r}}{8 \pi}
\end{gathered}
$$

and

$$
\begin{gathered}
\left(1-\frac{b}{r}\right)\left[\Phi^{\prime \prime}+\Phi^{\prime}\left(\Phi^{\prime}+\frac{1}{r}\right)\right]-\frac{\left(b^{\prime} r-b\right)}{2 r^{2}}\left(\Phi^{\prime}+\frac{1}{r}\right) \\
=p_{t}^{e f f}(r)=p\left(1+\frac{\rho}{\lambda}\right)+\frac{\rho^{2}}{2 \lambda}-\frac{\sigma_{t}}{8 \pi}
\end{gathered}
$$

Now if we claim that the solution (7) is also the solution of equations (14)-(16) (with matter components replaced by their effective components), then we must have

$$
\rho\left(1+\frac{\rho}{2 \lambda}\right)-\frac{\epsilon}{8 \pi}=\frac{d}{8 \pi r^{2}}
$$




$$
\begin{aligned}
& p\left(1+\frac{\rho}{\lambda}\right)+\frac{\rho^{2}}{2 \lambda}-\frac{\sigma_{r}}{8 \pi}=-\frac{1}{8 \pi r^{2}} \\
& p\left(1+\frac{\rho}{\lambda}\right)+\frac{\rho^{2}}{2 \lambda}-\frac{\sigma_{t}}{8 \pi}=\frac{(1-d)}{32 \pi r^{2}}
\end{aligned}
$$

Also the tracefree nature of $\varepsilon_{\mu}^{\nu}$ gives

$$
-\epsilon+\sigma_{r}+2 \sigma_{t}=0
$$

Hence we have four algebraic equations (17)-(20) containing five unknowns viz. $\rho, p, \epsilon, \sigma_{r}$ and $\sigma_{t}$. In the following, we shall present exact solutions for various assumptions:

\section{Case-I: $\quad \underline{\sigma_{t}=0}$}

The complete solution is

$$
\begin{aligned}
& \rho=\lambda\left[\sqrt{1+\frac{2 \mu}{\lambda r^{2}}}-1\right], \quad \mu=\frac{5+3 d}{32 \pi} \\
& p=\frac{\rho-\frac{1+d}{8 \pi r^{2}}}{\sqrt{1+\frac{2 \mu}{\lambda r^{2}}}} \\
& \rho+p=\frac{3+d}{16 \pi r^{2} \sqrt{1+\frac{2 \mu}{\lambda r^{2}}}}>0 \\
& \epsilon=\sigma_{r}=\frac{5-d}{4 r^{2}}
\end{aligned}
$$

\section{Case-II: $\quad \underline{\sigma_{r}=0}$}

The explicit solution reads

$$
\left.\begin{array}{l}
\rho=\lambda\left[\sqrt{1-\frac{2 \nu}{\lambda r^{2}}}-1\right]<0, \quad \nu=\frac{5-3 d}{16 \pi} \\
p=\frac{\rho+\frac{3(1-d)}{16 \pi r^{2}}}{\sqrt{1-\frac{2 \nu}{\lambda r^{2}}}} \\
\rho+p=\frac{3 d-7}{16 \pi r^{2} \sqrt{1-\frac{2 \nu}{\lambda r^{2}}}}<0 \\
\epsilon=-\frac{(5-d)}{2 r^{2}} \\
\sigma_{t}=-\frac{(5-d)}{4 r^{2}}
\end{array}\right\}
$$

\section{Case-III: $\quad \underline{\epsilon=0}$}

Here the solution becomes

$$
\begin{aligned}
& \rho=\lambda\left[\sqrt{1+\frac{d}{4 \pi \lambda r^{2}}}-1\right] \\
& p=\frac{\rho-\frac{7 d+1}{48 \pi r^{2}}}{\sqrt{1+\frac{d}{4 \pi \lambda r^{2}}}} \\
& \rho+p=\frac{5 d-1}{48 \pi r^{2} \sqrt{1+\frac{d}{4 \pi \lambda r^{2}}}}>0 \\
& \sigma_{r}=-2 \sigma_{t}=\frac{5-d}{6 r^{2}}
\end{aligned}
$$

\section{Case-IV: $\quad \underline{p=0 \text { (dust) }}$}

The solution is given by

$$
\left.\begin{array}{l}
\rho=\frac{\lambda}{2}\left[1 \pm \sqrt{1-\frac{1+3 d}{4 \pi \lambda r^{2}}}\right] \\
\epsilon=-\frac{1+7 d}{4 r^{2}}+6 \pi \lambda\left[1 \pm \sqrt{1-\frac{1+3 d}{4 \pi \lambda r^{2}}}\right] \\
\sigma_{r}=\frac{3(1-d)}{4 r^{2}}+2 \pi \lambda\left[1 \pm \sqrt{1-\frac{1+3 d}{4 \pi \lambda r^{2}}}\right] \\
\sigma_{t}=-\frac{d+1}{2 r^{2}}+2 \pi \lambda\left[1 \pm \sqrt{1-\frac{1+3 d}{4 \pi \lambda r^{2}}}\right]
\end{array}\right\}
$$

Case-V: $\quad \underline{\sigma_{r}=\sigma_{t}=\sigma(\text { say })}$

Then from equations (11) and (12), we must have

$$
d=5 \text { i.e, } \omega_{1}=1 / 5 \text { and } \omega_{2}=-1 / 5
$$

So the effective pressure on the brane becomes isotropic and we have got three equations viz.

$$
\begin{gathered}
\rho\left(1+\frac{\rho}{2 \lambda}\right)-\frac{\epsilon}{8 \pi}=\frac{5}{8 \pi r^{2}} \\
p\left(1+\frac{\rho}{\lambda}\right)+\frac{\rho^{2}}{2 \lambda}-\frac{\sigma}{8 \pi}=-\frac{1}{8 \pi r^{2}}
\end{gathered}
$$

and

$$
\epsilon=3 \sigma
$$

with four unknowns $\rho, p, \epsilon$ and $\sigma$. Let us assume the equation of state for brane matter as $p=\omega \rho$. Then we have the solution

$\rho=\frac{\lambda}{2}\left(\frac{1-3 \omega}{1+3 \omega}\right)\left[1 \pm \sqrt{1-\frac{4(1+3 \omega)}{\pi \lambda r^{2}(1-3 \omega)^{2}}}\right],\left(\omega \neq \frac{1}{3}\right)$ 
Further, for conformally flat bulk, $\varepsilon_{\mu \nu} \equiv 0$ i.e, $\epsilon=\sigma_{r}=\sigma_{t}=0$, and we have

$$
\left.\begin{array}{l}
\rho=\lambda\left[\sqrt{1+\frac{5}{4 \pi \lambda r^{2}}}-1\right] \\
p=\frac{\rho-\frac{3}{4 \pi r^{2}}}{\sqrt{1+\frac{5}{4 \pi \lambda r^{2}}}} \\
\text { with } \quad \rho+p=\frac{1}{2 \pi r^{2} \sqrt{1+\frac{5}{4 \pi \lambda r^{2}}}}>0
\end{array}\right\}
$$

In the present work, we have shown that a wormhole in Einstein gravity can be considered also as a wormhole in brane scenario. Here we have shown that the wormhole solution is possible for various matter distribution on the brane. It is interesting to note that, although the effective matter distribution violates the null energy condition, but the brane matter itself does not violate the null energy condition [11] in general. Except for Case-II (i.e, $\sigma_{r}=0$ ), in all other cases, the matter distribution obeys null energy condition. However, Case-II is a peculiar one as energy density is negative here. One may interprete such matter as ghost scalar field [17] or tachyonic matter field on the brane. In cases IV and V, where matter in the brane is of the form of dust or a perfect fluid with linear equation of state, the solutions obtained are similar to those presented by Lobo [11] [see eqns. (33)-(36) and (42)]. In solution (24), both the signs are possible as explicitly discussed in [11]. the solution (28) is valid for $\omega<1 / 3$ (otherwise $\rho$ will be negative), but if $\omega$ is associated to the phantom era $(\omega<-1 / 3)$, then we have positive energy density only for the negative sign within the square bracket. Therefore, instead of solving the complicated non linear second order differential equation to obtain wormhole solution, we have assumed the solution and obtained the possible matter distribution on the brane. For future work, we shall extend this idea for various cosmological solutions on the brane.

\section{Acknowledgement:}

The work has been done during a visit to IUCAA under the associateship programme. The authors gratefully acknowledge the warm hospitality and facilities of work at IUCAA. Also T.B is thankful to CSIR, Govt. of India, for awarding Junior Research Fellowship.
[1] M. S. Moris and K. S. Thorne, Am.J.Phys. 56, 396 (1988).

[2] M. S. Visser, Lorentzian Wormholes: From Einstein to Hawking (AIP Press, New York, 1995). See also M. S. Visser and D. Hochberg, "Geometric wormhole throats", gr-qc/9710001.

[3] A. Reiss et al., Astron.J. 116, 1009 (1998).

[4] S. J. Perlmutter et al., Astrophys.J. 517, 565 (1999).

[5] D. Hochberg and M. Visser, Phys.Rev.D 58, 044021 (1998).

[6] R. R. Caldwell, Phys.Lett.B 545, 23 (2002).

[7] L. Randall and R. Sundrum, Phys.Rev.Lett. 83, 3370 (1999).

[8] L. Randall and R. Sundrum, Phys.Rev.Lett. 83, 4690 (1999).

[9] R. Maartens, "Geometry and dynamics of the brane world", gr-qc/0101059 D. Langlois, "Gravitation and cosmology in a brane universe", gr-qc/0207047.
[10] K. A. Bronnikov and Sung-Won Kim, Phys.Rev.D 67, 064027 (2003).

[11] F. S. N. Lobo, Phys.Rev.D 75, 064027 (2007).

[12] O. B. Zaslavskii, Phys.Rev.D 72, 061303(R) (2005).

[13] F. S. N. Lobo, Phys.Rev.D 75, 024023 (2007); 73, 064028 (2006); 71, 124022 (2005); 71, 084011 (2005); J. P. S. Lemos, F. S. N. Lobo and S. Q. de Oliveira, Phys.Rev.D 68, 064004 (2003).

[14] S. V. Sushkov, Phys.Rev.D 71, 043520 (2005).

[15] T. Shiromizu, K. I. Maeda and M. Sasaki, Phys.Rev.D 62, 024012 (2000).

[16] R. Maartens, Living Rev. Relativity 7, 7 (2004).

[17] K. A. Bronnikov, Acta Phys.Pol.B 4, 251 (1973); H. Ellis, J.Math.Phys.(N.Y.) 14, 104 (1973); S. V. Sushkov and S. W. Kim, Gen.Relativ. Gravit. 36, 1671 (2004). 